\title{
Design and Implementation of MPICH2 over InfiniBand with RDMA Support*
}

\author{
Jiuxing Liu ${ }^{\dagger} \quad$ Weihang Jiang ${ }^{\dagger} \quad$ Pete Wyckoff ${ }^{\ddagger} \quad$ Dhabaleswar K. Panda $^{\dagger}$ \\ David Ashton $^{* *} \quad$ Darius Buntinas $^{* *} \quad$ William Gropp ${ }^{* *} \quad$ Brian Toonen $^{* *}$ \\ ${ }^{\dagger}$ Computer and Information Science, The Ohio State University, Columbus, OH 43210 \\ \{liuj, jiangw, panda\}@cis.ohio-state.edu \\ $\ddagger$ Ohio Supercomputer Center, 1224 Kinnear Road, Columbus, OH 43212 \\ pw@osc.edu
*** Mathematics and Computer Science Division, Argonne National Laboratory, Argonne, IL 60439 \{ashton, buntinas, gropp, toonen\}@mcs.anl.gov

\begin{abstract}
For several years, MPI has been the de facto standard for writing parallel applications. One of the most popular MPI implementations is MPICH. Its successor, MPICH2, features a completely new design that provides more performance and flexibility. To ensure portability, it has a hierarchical structure based on which porting can be done at different levels.

In this paper, we present our experiences designing and implementing MPICH2 over InfiniBand. Because of its high performance and open standard, InfiniBand is gaining popularity in the area of high-performance computing. Our study focuses on optimizing the performance of MPI-1 functions in MPICH2. One of our objectives is to exploit Remote Direct Memory Access (RDMA) in Infiniband to achieve high performance. We have based our design on the RDMA Channel interface provided by MPICH2, which encapsulates architecture-dependent communication functionalities into a very small set of functions.

Starting with a basic design, we apply different optimizations and also propose a zero-copy-based design. We characterize the impact of our optimizations and designs using microbenchmarks. We have also performed an applicationlevel evaluation using the NAS Parallel Benchmarks. Our

*This research is supported in part by Department of Energy's Grant \#DE-FC02-01ER25506, a grant from Sandia National Laboratories, a grant from Intel Corporation, National Science Foundation's grants \#CCR0204429 and \#CCR-0311542, and by the Mathematical, Information, and Computational Sciences Division subprogram of the Office of Advanced Scientific Computing Research, Office of Science, U.S. Department of Energy, under Contract W-31-109-ENG-38.
\end{abstract}

optimized MPICH2 implementation achieves $7.6 \mu$ s latency and $857 \mathrm{MB} / \mathrm{s}$ bandwidth, which are close to the raw performance of the underlying InfiniBand layer. Our study shows that the RDMA Channel interface in MPICH2 provides a simple, yet powerful, abstraction that enables implementations with high performance by exploiting RDMA operations in InfiniBand. To the best of our knowledge, this is the first high-performance design and implementation of MPICH 2 on InfiniBand using RDMA support.

\section{Introduction}

During the past ten years, the research and industry communities have proposed and implemented user-level communication systems to address some of the problems associated with traditional networking protocols. The Virtual Interface Architecture (VIA) [6] was proposed to standardize these efforts. More recently, the InfiniBand Architecture [8] has been introduced, which combines storage I/O with interprocess communication.

In addition to send and receive operations, InfiniBand architecture supports Remote Direct Memory Access (RDMA). RDMA operations enable direct access to the address space of a remote process. These operations introduce new opportunities and challenges in designing communication protocols.

In the area of high-performance computing, MPI [23] has been the de facto standard for writing parallel applications. After the original MPI standard (MPI-1), an enhanced 
standard (MPI-2) [17] was introduced, which includes features such as dynamic process management, one-sided communication, and I/O. MPICH [7] is one of the most popular MPI-1 implementations. Recently, work has begun to create MPICH2 [1], which aims to support both MPI-1 and MPI-2 standards. It features a completely new design that provides better performance and flexibility than its predecessor. MPICH2 is also very portable and provides mechanisms that make it easy to retarget MPICH2 to new communication architectures such as InfiniBand.

In this paper, we present our experiences in designing and implementing MPICH2 over InfiniBand using RDMA operations. Although MPICH2 supports both MPI-1 and MPI-2, our study focuses on optimizing the performance of MPI-1 functions. We have based our design on the RDMA Channel interface provided by MPICH2, which encapsulates architecture-dependent communication functionalities in a small set of functions. Despite its simple interface, we have shown that the RDMA Channel does not prevent one from achieving high performance. In our testbed, our MPICH2 implementation achieves $7.6 \mu$ s latency and $857 \mathrm{MB} / \mathrm{s}$ peak bandwidth, which are quite close to the raw performance of the InfiniBand platform. We have also evaluated our designs using the NAS Parallel Benchmarks [25]. Overall, we have demonstrated that the RDMA Channel interface is a simple, yet powerful, abstraction that makes it possible to design high-performance MPICH2 implementations with less development effort.

In our design, communication between processes is done exclusively using RDMA operations. Our design starts with an emulation of a shared-memory-based implementation. Then we introduce various optimization techniques to improve its performance. To evaluate the impact of each optimization, we use latency and bandwidth microbenchmarks. We also propose a zero-copy design for large messages. Our results show that with piggybacking and zero-copy optimizations for large messages, our design achieves very good performance.

The remainder of the paper is organized as follows. In Section 2, we provide an introduction to InfiniBand and its RDMA operations. In Section 3, we present an overview of MPICH2, its implementation structure, and the RDMA Channel interface. In Sections 4 and 5, we describe our designs and implementations. In Section 6, we compare our RDMA Channel-based design with another design based on a more complicated interface called $\mathrm{CH} 3$. In Section 7, we present an application level performance evaluation. In Section 8, we describe related work. In Section 9, we draw conclusions and briefly mention some future research directions.

\section{InfiniBand Overview}

The InfiniBand Architecture (IBA) [8] defines a switched network fabric for interconnecting processing nodes and $\mathrm{I} / \mathrm{O}$ nodes. It provides a communication and management infrastructure for interprocessor communication and I/O. In an InfiniBand network, processing nodes and I/O nodes are connected to the fabric by channel adapters (CAs). Channel adapters usually have programmable DMA engines with protection features. There are two kinds of channel adapters: host channel adapter (HCA) and target channel adapter (TCA). HCAs sit on processing nodes.

The InfiniBand communication stack consists of different layers. The interface presented by channel adapters to consumers belongs to the transport layer. A queue-based model is used in this interface. A queue pair in InfiniBand Architecture consists of two queues: a send queue and a receive queue. The send queue holds instructions to transmit data, and the receive queue holds instructions that describe where received data is to be placed. Communication operations are described in work queue requests (WQRs), or descriptors, and are submitted to the work queue. The completion of WQRs is reported through completion queues (CQs). Once a work queue element is finished, a completion queue entry is placed in the associated completion queue. Applications can check the completion queue to see whether any work queue request has been finished. InfiniBand also supports different classes of transport service. In this paper, we focus on the reliable connection (RC) service.

\subsection{RDMA Operations in InfiniBand Architec- ture}

InfiniBand Architecture supports both channel and memory semantics. In channel semantics, send/receive operations are used for communication. To receive a message, the programmer posts a receive descriptor that describes where the message should be put at the receiver side. At the sender side, the programmer initiates the send operation by posting a send descriptor.

In memory semantics, InfiniBand supports remote direct memory access (RDMA) operations, including RDMA write and RDMA read. RDMA operations are one sided and do not incur software overhead at the remote side. In these operations, the sender (initiator) starts RDMA by posting RDMA descriptors. A descriptor contains both the local data source addresses (multiple data segments can be specified at the source) and the remote data destination address. At the sender side, the completion of an RDMA operation can be reported through CQs. The operation is transparent to the software layer at the receiver side.

Since RDMA operations enable a process to access the address space of another process directly, they have raised 
some security concerns. In InfiniBand architecture, a keybased mechanism is used. A memory buffer must first be registered before it can be used for communication. Among other things, the registration generates a remote key. This remote key must be presented when the sender initiates an RDMA operation to access the buffer.

Compared with send/receive operations, RDMA operations have several advantages. First, RDMA operations themselves are generally faster than send/receive operations because they are simpler at the hardware level. Second, they do not involve managing and posting descriptors at the receiver side, which would incur additional overheads and reduce the communication performance.

\section{MPICH2 Overview}

MPICH [7] is developed at Argonne National Laboratory. It is one of the most popular MPI implementations. The original MPICH provides support for the MPI-1 standard. As a successor of MPICH, MPICH2 [1] aims to support not only the MPI-1 standard but also functionalities such as dynamic process management, one-sided communication, and MPI I/O, which are specified in the MPI-2 standard. However, MPICH2 is not merely MPICH with MPI-2 extensions. It is based on a completely new design, aiming to provide more performance, flexibility, and portability than the original MPICH. One of the notable features in the implementation of MPICH2 is that it can take advantage of RDMA operations if they are provided by the underlying interconnect. These operations can be used not only to support MPI-2 one-sided communication but also to implement normal MPI-1 communication. Although MPICH2 is still under development, beta versions are already available for developers. In the current version, all MPI-1 functions have been implemented. MPI- 2 functions are not completely supported yet. In this paper, we mainly focus on the MPI-1 part of MPICH2.

\subsection{MPICH2 Implementation Structure}

One of the objectives in MPICH2 design is portability. To facilitate porting MPICH2 from one platform to another, MPICH2 uses ADI3 (the third generation of the Abstract Device Interface) to provide a portability layer. ADI3 is a full-featured abstract device interface and has many functions, so it is not a trivial task to implement all of them. To reduce the porting effort, $\mathrm{MPICH} 2$ introduces the $\mathrm{CH} 3$ interface. $\mathrm{CH} 3$ is a layer that implements the ADI3 functions and provides an interface consisting of only a dozen functions. A "channel" implements the $\mathrm{CH} 3$ interface. Channels exist for different communication architectures such as TCP sockets and, SHMEM. Because only a dozen functions are associated with each channel interface, it is easier to implement a channel than the ADI3 device.

To take advantage of architectures with globally shared memory or RDMA capabilities and to further reduce the porting overhead, MPICH2 introduces the RDMA Channel which implements the $\mathrm{CH} 3$ interface. The RDMA Channel interface only contains five functions. We will discuss the details of the RDMA Channel interface in the next subsection.

The hierarchical structure of MPICH2, as shown in Figure 1 gives much flexibility to implementors. The three interfaces (ADI3, CH3, and the RDMA Channel interface) provide different trade-offs between communication performance and ease of porting.

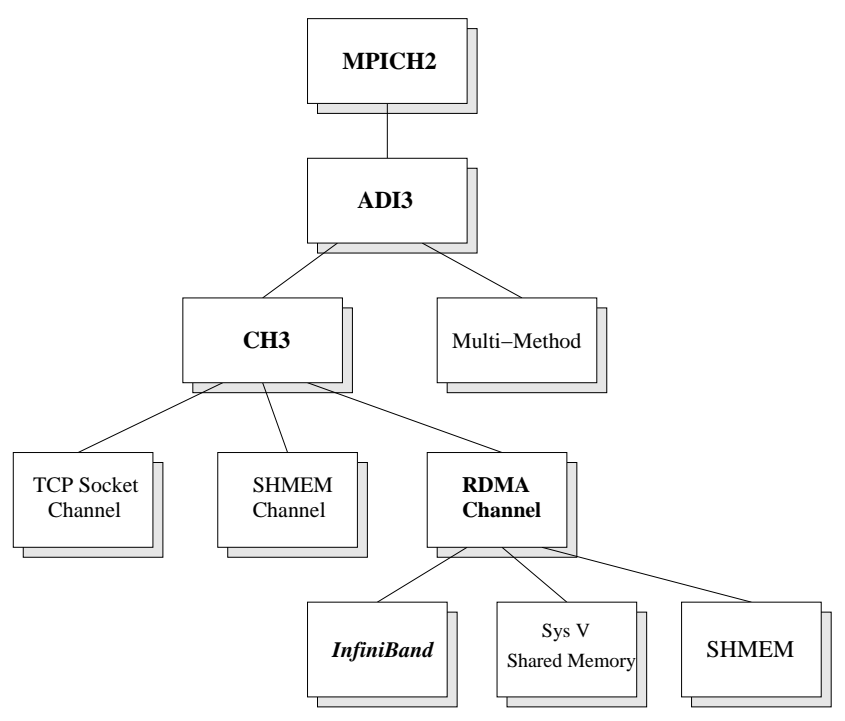

Figure 1. MPICH2 Implementation Structure

\subsection{MPICH2 RDMA Channel Interface}

The MPICH2 RDMA Channel interface is designed specifically for architectures with globally shared memory or RDMA capabilities. It contains five functions, among which only two are central to communication. (Other functions deal with process management, initialization, and finalization.) These two functions are called put (write) and get (read).

Both put and get functions accept a connection structure and a list of buffers as parameters. They return to the caller the number of bytes that have been successfully put or gotten. If the number of bytes completed is less than the total length of buffers, the caller will retry the same get or put operation later.

Figure 2 illustrates the semantics of put and get. Logically, a pipe is shared between the sender and the receiver. The put operation writes to the pipe, and the get operation 
reads from it. The data in the pipe is consumed in FIFO order. Both operations are nonblocking in the sense that they return immediately with the number of bytes completed, instead of waiting for the entire operation to finish. We note that put and get are different from RDMA write and RDMA read in InfiniBand. While RDMA operations in InfiniBand are one sided, put and get in the RDMA Channel interface are essentially two-sided operations.

Put and get operations can be implemented on architectures with globally shared memory in a straightforward manner. Figure 3 shows one example. In this implementation, a shared buffer (organized logically as a ring) is placed in shared memory, together with a head pointer and a tail pointer. The put operation copies the user buffer to the shared buffer and adjusts the head pointer. The get operation involves reading from the shared buffer and adjusting the tail pointer. In the case of buffer overflow or underflow (detected by comparing head and tail pointers), the operations return immediately, and the caller will retry them.

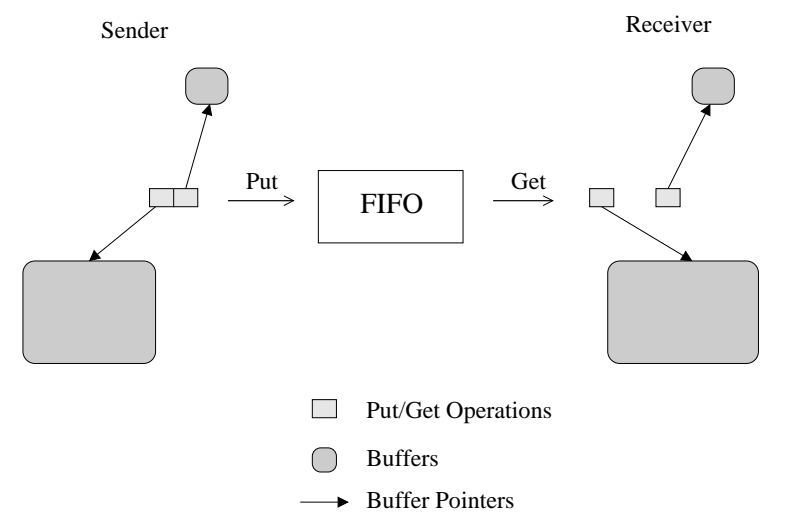

Figure 2. Put and Get Operations

Working at the RDMA Channel interface level is better than writing a new $\mathrm{CH} 3$ or ADI3 implementation for many reasons:

1. Improvements done at this level can affect all sharedmemory-like transports such as globally shared memory, RDMA over IP, Quadrics, and Myrinet.

2. Other protocols on InfiniBand need efficient processing, including one-sided communication in MPI-2, DSM systems, and parallel file systems. The RDMA Channel interface can potentially be used also for them.

3. Designing proper interfaces to similar systems improves performance and portability in general.

In collaboration, the OSU and ANL teams are also currently working together to design an improved interface that can benefit communication systems in general.

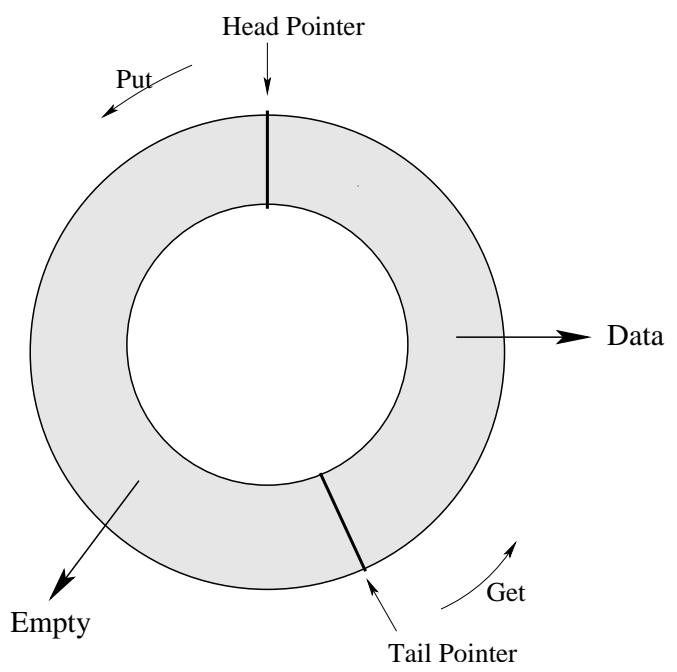

Figure 3. Put and Get Implementation with Globally Shared Memory

\section{Designing and Optimizing MPICH2 over InfiniBand}

In this section, we present several different designs of MPICH2 over InfiniBand based on the RDMA Channel interface. We first start with a basic design that resembles the scheme described in Figure 3 Then we apply various optimization techniques to improve its performance. In this section, the designs are evaluated by using microbenchmarks such as latency and bandwidth. We show that by taking advantage of RDMA operations in InfiniBand, we can achieve not only low latency for small messages but also high bandwidth for large messages using the RDMA Channel interface. In Section 5, we present a zero-copy design.

\subsection{Experimental Testbed}

Our experimental testbed consists of a cluster system with 8 SuperMicro SUPER P4DL6 nodes. Each node has dual Intel Xeon $2.40 \mathrm{GHz}$ processors with a 512K L2 cache and a $400 \mathrm{MHz}$ front side bus. The machines are connected by Mellanox InfiniHost MT23108 DualPort 4X HCA adapter through an InfiniScale MT43132 Eight 4x Port InfiniBand Switch. The HCA adapters work under the PCI-X 64-bit 133MHz interfaces. We used the Linux Red Hat 7.2 operating system with 2.4 .7 kernel. The compilers we used were GNU GCC 2.96 and GNU FORTRAN 0.5.26.

\subsection{Basic Design}

In Figure 3 we illustrated how the RDMA Channel interface can be implemented on shared-memory architectures. 
In a cluster connected by InfiniBand, however, there is no physically shared memory. In our basic design, we use RDMA write operations provided by InfiniBand to emulate this scheme.

We put the shared-memory buffer in the receiver's main memory. This memory is registered and exported to the sender. Therefore, it is accessible to the sender through RDMA operations. To avoid the relatively high cost of registering user buffers for sending every message, we also use a preregistered buffer at the sender that is the same size as the shared buffer at the receiver. User data is first copied into this buffer and then sent out. Head and tail pointers also need to be shared between the sender and the receiver. Since they are used frequently at both sides, we use replication to prevent polling through the network. For the tail pointer, a master copy is kept at the receiver, and a replica is kept at the sender. For the head pointer, a master copy is kept at the sender, and a replica is kept at the receiver. For each direction of every connection, the associated "shared" memory buffer, head and tail pointers are registered during initialization, and their addresses and remote keys are exchanged.

At the sender, the put operation is implemented as follows:

1. Use local copies of head and tail pointers to decide how much empty space is available.

2. Copy user buffer to the preregistered buffer.

3. Use RDMA write operation to write the data to the buffer at the receiver side.

4. Adjust the head pointer based on the amount of data written.

5. Use another RDMA write to update the remote copy of head pointer.

6. Return the number of bytes written.

At the receiver, the get operation is implemented in the following way:

1. Check local copies of head and tail pointers to see whether there is new data available.

2. Copy the data from the shared memory buffer to user buffer.

3. Adjust the tail pointer based on the amount of data that has been copied.

4. Use an RDMA write to update the remote copy of tail pointer.

5. Return the number of bytes successfully read.
We note that copies of head and tail pointers are not always consistent. For example, after a sender adjusts its head pointer, it uses RDMA write to update the remote copy at the receiver. Therefore, the head pointer at the receiver is not up to date until the RDMA write finishes. However, this inconsistency does not affect the correctness of the scheme; it merely prevents the receiver from reading new data temporarily. Similarly, inconsistency of tail pointer may prevent the sender from writing to the shared buffer. But eventually the pointers will become up to date, and the sender or the receiver will be able to make progress.

\subsubsection{Performance of the Basic Design}

We use latency and bandwidth tests to evaluate the performance of our basic design. The latency test is conducted in a ping-pong fashion, and the results are derived from roundtrip time. In the bandwidth test, a sender keeps sending back-to-back messages to the receiver until it has reached a predefined window size $W$. Then it waits for these messages to finish and send out another $W$ messages. The results are derived from the total test time and the number of bytes sent.

Figures 4 and 5 show the results. Our basic design achieves a latency of $18.6 \mu$ s for small messages and a bandwidth of $230 \mathrm{MB} / \mathrm{s}$ for large messages. (Unless stated otherwise, the unit MB in this paper is an abbreviation for $10^{6}$ bytes, not $2^{20}$ bytes.) These numbers are much worse than the raw performance numbers achievable by the underlying InfiniBand layer (5.9 $\mu$ s latency and $870 \mathrm{MB} / \mathrm{s}$ bandwidth).

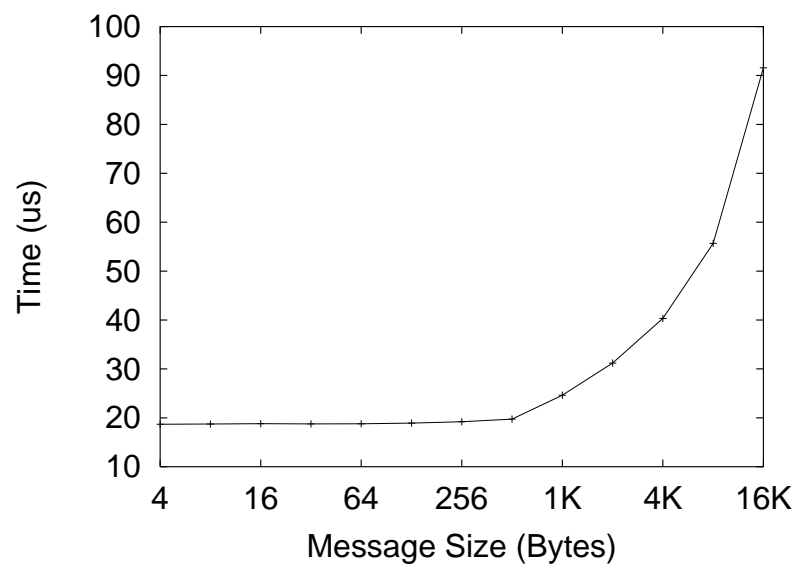

Figure 4. MPI Latency for Basic Design

A careful look at the basic design reveals many inefficiencies. For example, a matching pair of send and receive operations in MPI require three RDMA write operations to take place: one for transfer of data, and two for updating head and tail pointers. These not only increase latency and host overhead but also generate unnecessary network traffic. 


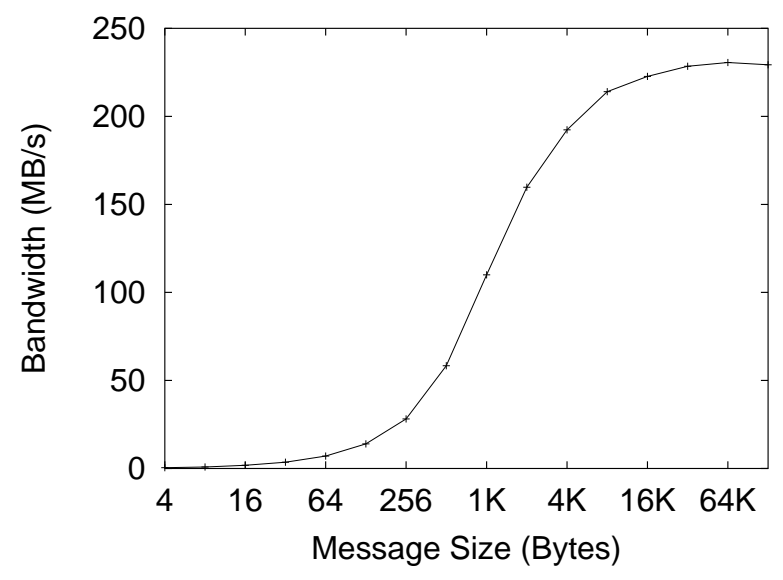

Figure 5. MPI Bandwidth for Basic Design

For large messages, the basic scheme leads to two extra memory copies. The first one is from user buffer to the preregistered buffer at the sender side. The second one is from the shared buffer to user buffer at the receiver side. These memory copies consume resources such as memory bandwidth and CPU cycles. To make matters worse, in the basic design the memory copies and communication operations are serialized. For example, a sender first copies the whole message (or part of the message if it cannot fit itself in the empty space of the preregistered buffer). Then it initiates RDMA write to transfer the data. This serialization of copying and RDMA write greatly reduces the bandwidth for large messages.

\subsection{Optimization with Piggybacking Pointer Up- dates}

Our first optimization targeted to avoid separate head and tail pointer updates whenever possible. The technique we used is piggybacking, which combines pointer updates with data transfer.

At the sender side, we combine data and the new value of head pointer into a single message. To help the receiver detect the arrival of the message, we attach the size with the message and put two flags at the beginning and the end of the message. The receiver detects arrival of the new message by polling on the flags. To avoid possible situations where the buffer content happens to have the same value as the flag, we divide the shared buffer into fixed-sized chunks. Each message uses a different chunk. In this way, the situations can be handled by using two polling flags, or "bottom fill." Similar techniques have been used in [16, 21].

At the receiver side, instead of using RDMA write to update the remote tail pointer each time data has been read, we delay the updates until the free space in the shared buffer drops below a certain threshold. If messages are sent from the receiver to the sender, the pointer value is attached with the message, and no extra message is used to transfer pointer updates. If no messages are sent from the receiver to the sender, eventually we will explicitly send the updates by using an extra message. The sender updates its pointer after receiving this message. Even in this case, however, the traffic can be reduced because several consecutive updates of the tail pointer can be sent by using only one message.

The use of piggybacking and delayed pointer updates can greatly improve the performance of small message. From Figure 6 we see that the latency is reduced from $18.6 \mu$ s to $7.4 \mu$ s. Figure 7 shows that the optimization also improves bandwidth for small messages.

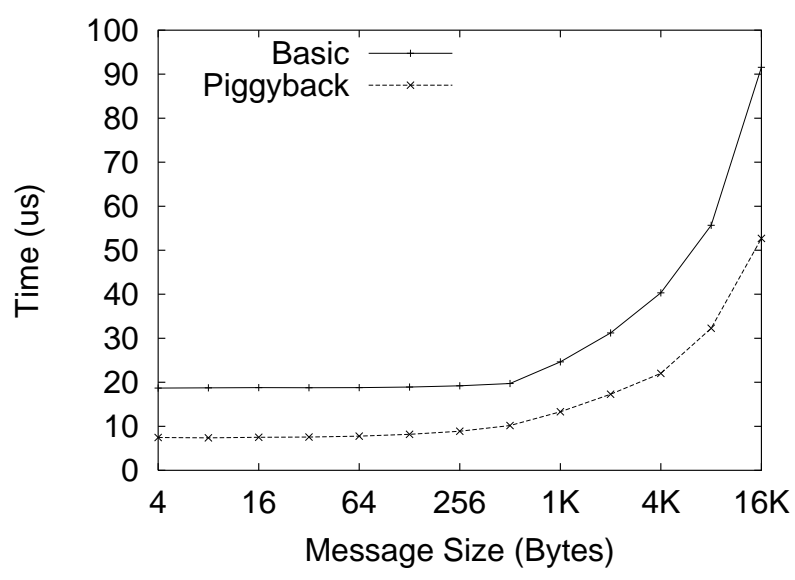

Figure 6. MPI Small-Message Latency with Piggybacking

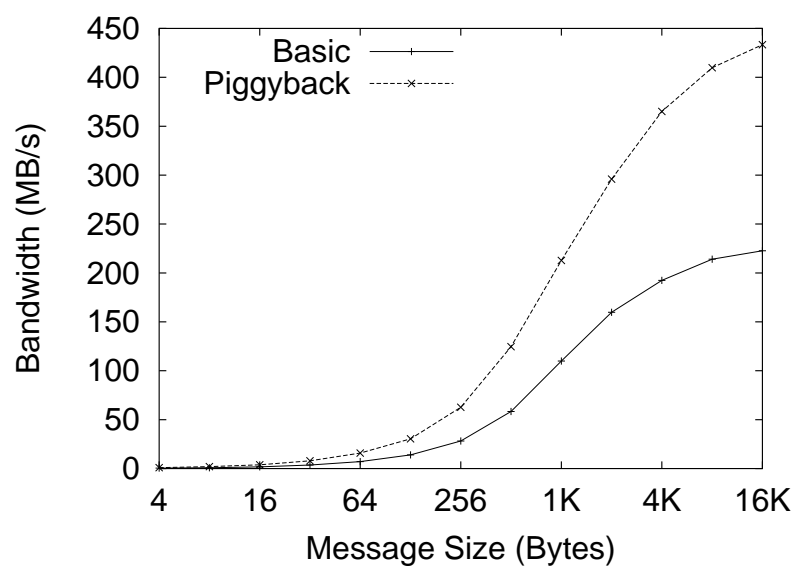

Figure 7. MPI Small-Message Bandwidth with Piggybacking 


\subsection{Optimization with Pipelining of Large Mes- sages}

As we have discussed, our basic design suffers from serialization of memory copies and RDMA writes. A better solution is to use pipelining to overlap memory copies with RDMA write operations.

In our piggybacking optimization, we divide the sharedmemory buffer into small chunks. When sending and receiving large messages, we need to use more than one such chunks. At the sender side, instead of starting RDMA writes after copying all the chunks, we initiate the RDMA transfer immediately after copying each chunk. In this way, the RDMA operation can be overlapped with the copying of the next chunk. Similarly, at the receiver side we start copying from the shared buffer to the user buffer immediately after a chunk is received. In this way, the receive RDMA operations can be overlapped with the copying.

Figure 8 compares the bandwidth of the pipelining scheme with the basic scheme. (Piggybacking is also used in the pipelining scheme.) We can see that pipelining combined with piggybacking has greatly improved MPI bandwidth. The peak bandwidth has been increased from $230 \mathrm{MB} / \mathrm{s}$ to over $500 \mathrm{MB} / \mathrm{s}$. This result is still not satisfying, however, because InfiniBand is able to deliver bandwidth up to $870 \mathrm{MB} / \mathrm{s}$.

To investigate the performance bottleneck, we have conducted memory copy tests in our testbed. We have found that memory copy bandwidth is less than $800 \mathrm{MB} / \mathrm{s}$ for large messages. In our MPI bandwidth tests, with RDMA write operations and memory copies both using the memory bus, the bandwidth achievable at the application level is even less. Therefore, the memory bus clearly becomes a performance bottleneck for large messages because of the extra memory copies.

In the pipelining optimization, it is important that we balance each stage of the pipeline so that we can get maximum throughput. One parameter we can change to balance pipeline stages is the chunk size, or how much data we copy each time for a large message. Figure 9 shows MPI bandwidth for different chunk sizes for the pipelining optimization. We observe that MPI does not give good performance when the chunk size is either too small (1K Bytes) or too large (32K Bytes). MPI performs comparably for chunk sizes of $2 \mathrm{~K}$ to $16 \mathrm{~K}$ Bytes. In all remaining tests, we have chosen a chunk size of 16K Bytes.

\section{Zero-Copy Design}

As we have discussed in the preceding section, it is desirable to avoid memory copies for large messages. In this section, we describe a zero-copy design for large messages based on the RDMA Channel interface.

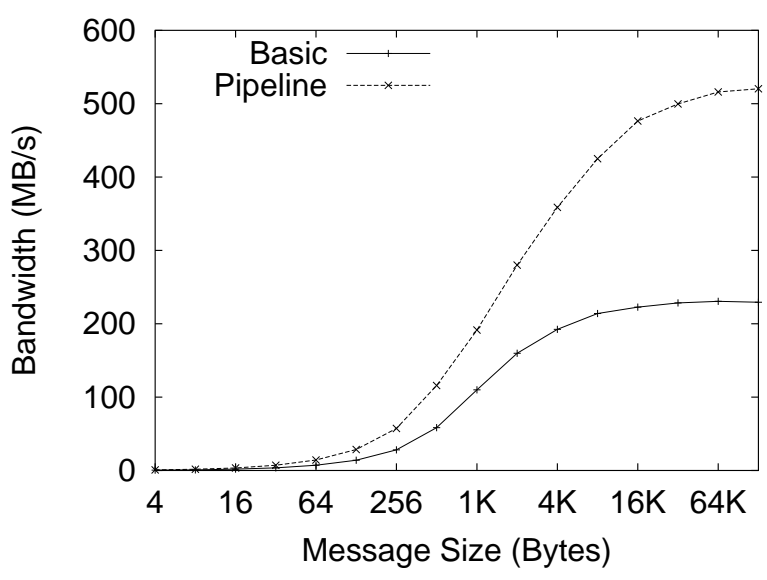

Figure 8. MPI Bandwidth with Pipelining

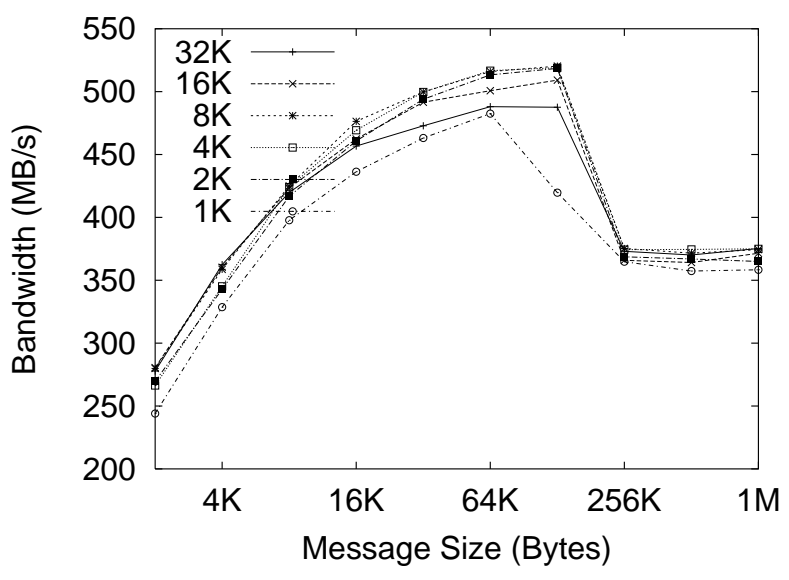

Figure 9. MPI Bandwidth with Pipelining (Different Chunk Sizes) 
In our new design, small messages are still transferred by using RDMA write, similar to the piggybacking scheme. For large messages, however, RDMA read, instead of RDMA write, is used for data transfer. The basic idea of our zero-copy design is to let the receiver "pull" the data directly from the sender using RDMA read.

For each connection, shared buffers are still used for transferring small messages. However, the data for large messages is not transferred through the shared buffer. At the sender, when the put function is called, it checks the user buffer and decides whether to use zero-copy or not, based on the buffer size. If zero-copy is not used, the message is sent through the shared buffer as discussed before. If zero-copy is used, the function registers the user buffer, constructs a special packet that contains information about the user buffer such as address, size, and remote key, then sends the special packet by using RDMA write through the shared buffer. The put function returns a value of 0 , at this stage, because no data has been transferred yet. Subsequent calls to put also return 0 until all of the data has been transferred, and the operation has completed. Once the operation has completed, put will return the number of bytes transferred.

When the packet arrives at the other side and the get function is called, the receiver checks the shared buffer and processes all the packets in order. If a packet is a data packet, the data is copied to the user buffer. If it is a special packet, the user buffer is registered, and an RDMA read operation is issued to fetch the data from the remote side directly to the user buffer. After initiating the RDMA read, the get function returns with a value of 0 , because the operation is still in progress. When the RDMA read is finished, calling the get function leads to an acknowledgment packet being sent to the sender. The get function then returns the number of bytes successfully transferred. When the acknowledgment packet is received at the sender side, the sender deregisters the user buffer, completing the operation, and the next call to the put function will return the number of bytes transferred. The zero-copy process is illustrated in Figure 10

In the current InfiniBand implementation, memory registration and deregistration are expensive operations. To reduce the number of registrations and deregistrations, we have implemented a registration cache [24]. The basic idea is to delay the deregistration of user buffers and put them into a cache. If the same buffer is reused later, its registration information can be fetched directly from the cache instead of going through the expensive registration process. Deregistration happens only when there are too many registered user buffers.

We note that the effectiveness of registration cache depends on buffer reuse patterns of applications. If applications rarely reuse buffers for communication, registration overhead cannot be avoided most of the time. Fortunately,

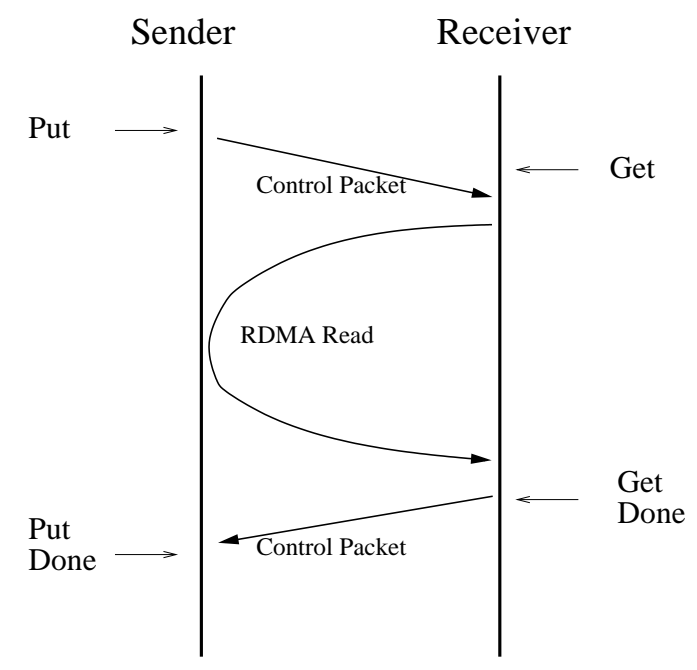

Figure 10. Zero-Copy Design

our previous study with the NAS Parallel Benchmarks [15] has demonstrated that buffer reuse rates are very high in these applications.

We compare the bandwidth of the pipelining design and the zero-copy design in Figure 11 We observe that zero-copy greatly improves the bandwidth for large messages. We achieve a peak bandwidth of $857 \mathrm{MB} / \mathrm{s}$, which is quite close to the peak bandwidth at the InfiniBand level $(870 \mathrm{MB} / \mathrm{s})$. We also see that as a result of cache effect, bandwidth for large messages drops for the pipelining design. Because of the extra overhead in the implementation, the zero-copy design slightly increases the latency for small messages, which is now around $7.6 \mu \mathrm{s}$.

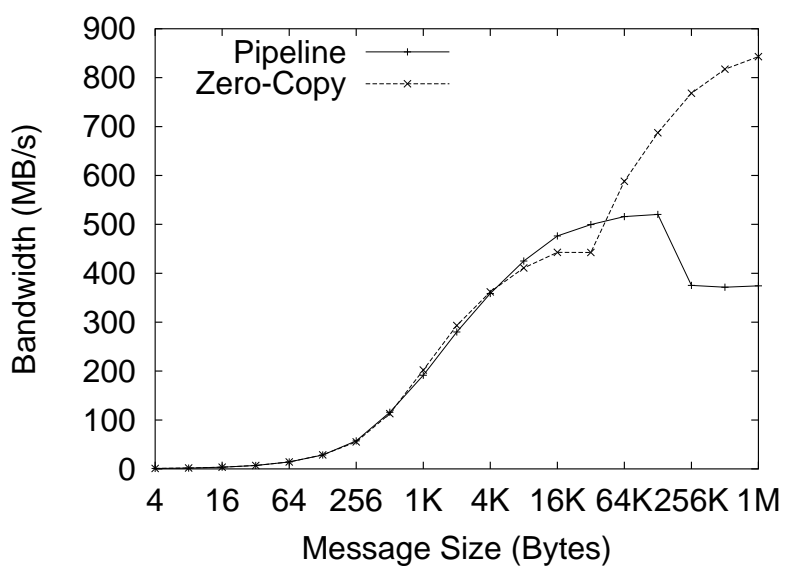

Figure 11. MPI Bandwidth with Zero-Copy and Pipelining

Our zero-copy implementation uses RDMA read operations, which let the receiver "pull" data from the sender. 
An alternative is to use RDMA write operations and let the sender "push" data to the receiver. Before the sender can push the data, the receiver has to use special packets to advertise availability of new receive buffers. Therefore, this method can be very efficient if the get operations are called before the corresponding put operations. In the current MPICH2 implementation, however, the layers above the RDMA Channel interface are implemented in such a way that get is always called after put for large messages. Therefore, we have chosen an RDMA read-based implementation instead of RDMA write.

\section{Comparing CH3 and RDMA Channel In- terface Designs}

The RDMA Channel interface in MPICH2 provides a simple way to implement MPICH2 in many communication architectures. In the preceding section, we showed that this interface does not prevent one from achieving good performance. Nor does it prevent zero-copy implementation for large messages. Our results showed that with various optimizations, we can achieve a latency of $7.6 \mu \mathrm{s}$ and a peak bandwidth of $857 \mathrm{MB} / \mathrm{s}$.

The CH3 interface is more complicated than the RDMA Channel interface. Therefore, porting it requires more effort. However, since $\mathrm{CH} 3$ provides more flexibility, it is possible to achieve better performance at this level.

To study the impact of different interfaces on MPICH2 performance, we have also done a $\mathrm{CH} 3$-level implementation. This implementation uses RDMA write operations for transferring large messages, as shown in Figure 12 Before transferring the message, a handshake happens between the sender and the receiver. User buffer at the receiver is registered and its information is sent to the sender through the handshake. The sender then uses RDMA write to transfer the data. A registration cache is also used in this implementation.

Figures 13 and 14 compare this implementation with our RDMA Channel-based zero-copy design using latency and bandwidth microbenchmarks. We see that the two implementations perform comparably for small and large messages. However, the $\mathrm{CH} 3$-based design outperforms the RDMA Channel-based design for mid-sized messages (32K to $256 \mathrm{~K}$ Bytes) in bandwidth.

Figure 15 shows the bandwidth of RDMA read and RDMA write at the InfiniBand VAPI level. (VAPI is the programming interface for our InfiniBand cards.) With the current VAPI implementation, RDMA write operations have a clear advantage over RDMA read for mid-sized messages. Therefore, the fact that $\mathrm{CH} 3$-based design outperforms RDMA Channel-based design for mid-sized messages is more the result of the raw performance difference between RDMA write and RDMA read than the designs themselves.

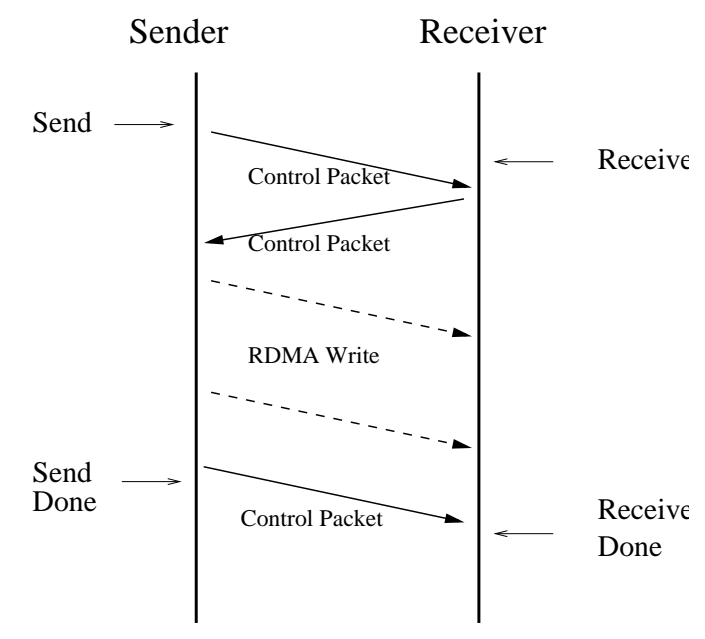

Figure 12. CH3 Zero-Copy with RDMA Write

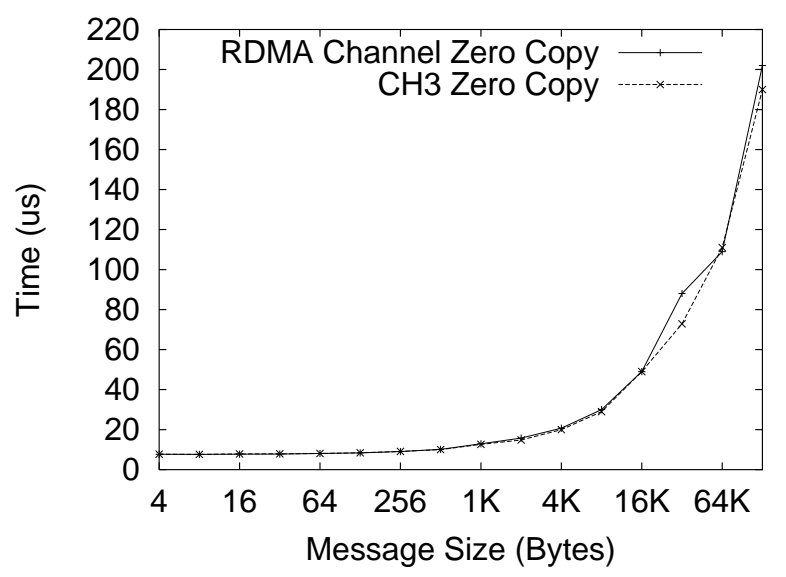

Figure 13. MPI Latency for $\mathrm{CH} 3$ Design and RDMA Channel Interface Design

\section{Application-Level Evaluation}

In this section, we carry out an application-level evaluation of our MPICH2 designs using NAS Parallel Benchmarks [25]. We run class A benchmarks on 4 nodes and class B benchmarks on 8 nodes. Benchmarks SP and BT require a square number of nodes. Therefore, their results are only shown for 4 nodes.

The results are shown in Figures 16 and 17 We have evaluated three designs: RDMA Channel implementation with pipelining for large messages (Pipelining), RDMA Channel implementation with zero-copy for large messages 


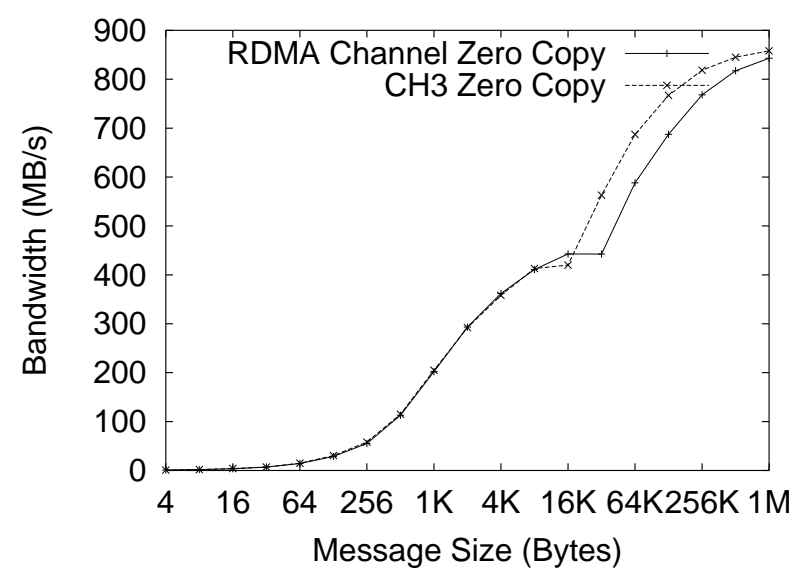

Figure 14. MPI Bandwidth for $\mathrm{CH} 3$ Design and RDMA Channel Interface Design

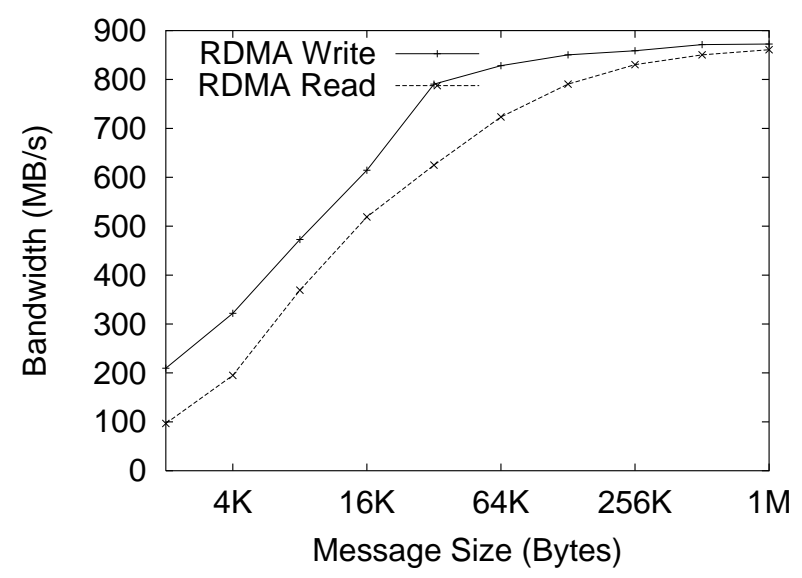

Figure 15. InfiniBand Bandwidth
(RDMA Channel), and CH3 implementation with zerocopy $(\mathrm{CH} 3)$. Although the performance difference of these three designs is not much, we observe that the pipelining design performs the worst in all cases. The RDMA Channelbased zero-copy design performs very close to the the $\mathrm{CH} 3-$ based zero-copy design. On average, the $\mathrm{CH} 3$-based design performs less than $1 \%$ better on both 4 nodes and 8 nodes.

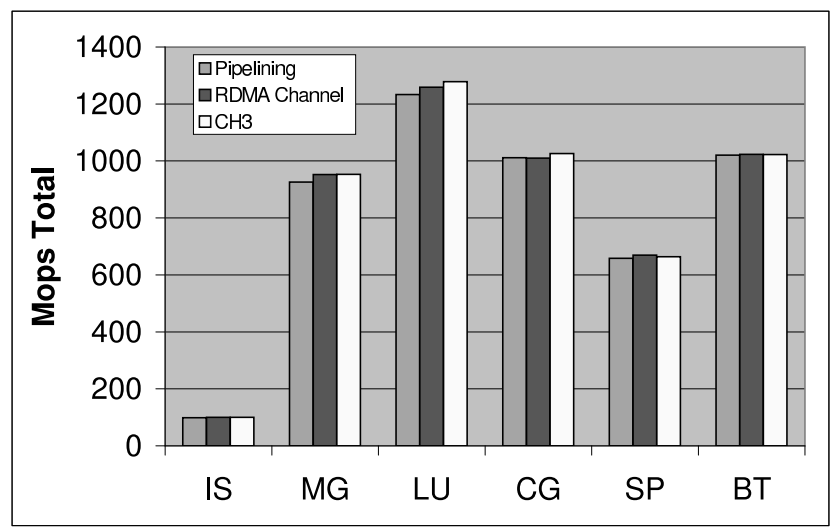

Figure 16. NAS Class A on 4 Nodes

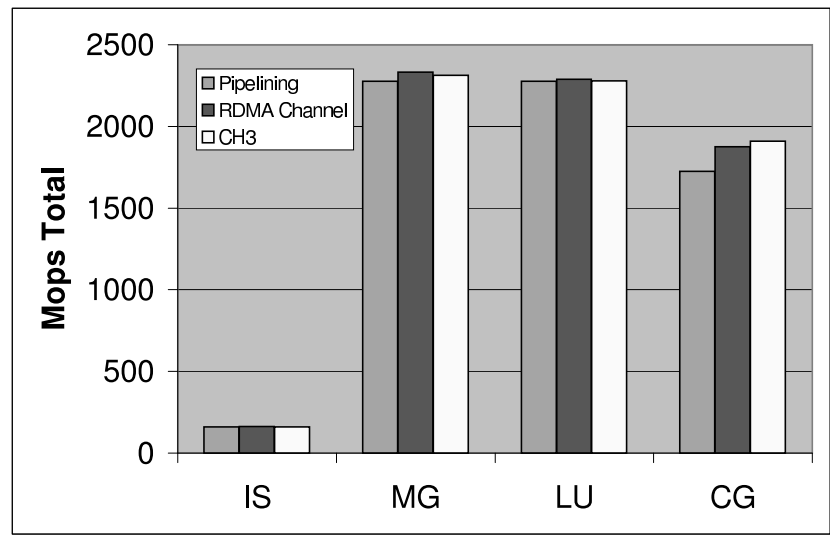

Figure 17. NAS Class B on 8 Nodes

\section{Related Work}

As the predecessor of MPICH2 and one of the most popular MPI implementations, MPICH supports a similar implementation structure as MPICH2. MPICH provides ADI2 (the second generation of Abstract Device Interface) and Channel interface. Various implementations exist based on these interfaces [3, 18, 20, 13]. Our MVAPICH implementation [16, which exploits RDMA write in InfiniBand, is based on the ADI2 interface.

Since MPICH2 is relatively new, there exists very little work describing its implementations on different architec- 
tures. In [2], a CH3-level implementation based on TCP/IP is described. Work in [22] presents an implementation $\mathrm{MPICH} 2$ over InfiniBand, also using the $\mathrm{CH} 3$ interface. However, in our paper, our focus is on the RDMA Channel interface instead of the $\mathrm{CH} 3$ interface. MPICH2 is designed to support both MPI-1 and MPI-2 standards. There have been studies about supporting the MPI- 2 standard, especially one-sided communication operations [5, 10]. To date, we have concentrated on supporting MPI-1 functions in MPICH2. We plan to explore the support of MPI-2 functions in the future.

Because of its high bandwidth and low latency, the InfiniBand Architecture has been used as the communication subsystem in a number of systems other than MPI, such as distributed shared-memory systems and parallel file systems [11, 14].

The RDMA Channel interface presents a stream-based abstraction somewhat similar to the traditional socket interface. There have been studies of how to implement userlevel socket interface efficiently over high-speed interconnects such as Myrinet, VIA, and Gigabit Ethernet [19 12 4]. Recently, Socket Direct Protocol (SDP) [9] has been proposed, which provides a socket interface over InfiniBand. The idea of our zero-copy scheme is similar to the Z-Copy scheme in SDP. However, there are also differences between the RDMA Channel interface and the traditional socket interface. For example, put and get functions in RDMA Channel interface are nonblocking, while functions in the traditional sockets are usually blocking. To support traditional socket interface, one has to make sure the same semantics are maintained. We do not have to deal with this issue for the RDMA Channel interface.

\section{Conclusions and Future Work}

In this paper, we present a study of using RDMA operations to implement MPICH2 over InfiniBand. Our work takes advantage of the RDMA Channel interface provided by MPICH2.

The RDMA Channel interface provides a very small set of functions to encapsulate the underlying communication layer on which the whole MPICH2 implementation is built. Consisting of only five functions, the RDMA Channel interface is easy to implement for different communication architectures. However, the question arises whether this abstraction is powerful enough that one can still achieve good performance.

Our study has shown that the RDMA Channel interface still provides the implementors much flexibility. With optimizations such as piggybacking, pipelining, and zero-copy, $\mathrm{MPICH} 2$ is able to deliver good performance to the application layer. For example, one of our designs achieves $7.6 \mu \mathrm{s}$ latency and $857 \mathrm{MB} / \mathrm{s}$ peak bandwidth, which come quite close to the raw performance of InfiniBand. In our study, we characterize the impact of each optimization by using latency and bandwidth microbenchmarks. We have also conducted an application-level evaluation using the NAS Parallel Benchmarks.

So far, our study has been restricted to a fairly small platform consisting of 8 nodes. In the future, we plan to use larger clusters to study various aspects of our designs regarding scalability. Another direction we are pursuing is to provide support for MPI-2 functionalities such as one-sided communication using RDMA and atomic operations in InfiniBand. We are also working on how to support efficient collective communication on top of InfiniBand.

\section{References}

[1] Argonne National Laboratory. MPICH2. http://www.mes. anl.gov/mpi/mpich2.

[2] D. Ashton, W. Gropp, R. Thakur, and B. Toonen. The CH3 Design for a Simple Implementation of ADI-3 for MPICH with a TCP-based Implementation. http://www.mcs.anl.gov/ mpi/mpich2/docs/tcpadi3.ps.

[3] O. Aumage, G. Mercier, and R. Namyst. MPICH/Madeleine: A True Multi-Protocol MPI for High-Performance Networks. In Proc. 15th International Parallel and Distributed Processing Symposium (IPDPS 2001), page 51, San Francisco, Apr. 2001.

[4] P. Balaji, P. Shivam, P. Wyckoff, and D. K. Panda. High Performance User Level Sockets over Gigabit Ethernet. In Proc. IEEE International Conference on Cluster Computing, 2002.

[5] S. Booth and F. E. Mourao. Single Sided MPI implementations for SUN MPI. In Supercomputing, 2000.

[6] D. Dunning, G. Regnier, G. McAlpine, D. Cameron, B. Shubert, F. Berry, A. Merritt, E. Gronke, and C. Dodd. The Virtual Interface Architecture. IEEE Micro, pages 66-76, March/April 1998.

[7] W. Gropp, E. Lusk, N. Doss, and A. Skjellum. A HighPerformance, Portable Implementation of the MPI Message Passing Interface Standard. Parallel Computing, 22(6):789828, 1996.

[8] InfiniBand Trade Association. InfiniBand Architecture Specification, Release 1.0, October 242000.

[9] InfiniBand Trade Association. Socket Direct Protocol Specification V1.0, 2002.

[10] J. Traff and H. Ritzdorf and R. Hempel. The Implementation of MPI-2 One-Sided Communication for the NEC SX. In Proc. Supercomputing, 2000.

[11] J. Wu and P. Wyckoff and D. K. Panda. PVFS over InfiniBand: Design and Performance Evaluation. In Proc. International Conference on Parallel Processing, 2003.

[12] J.-S. Kim, K. Kim, and S.-I. Jung. SOVIA: A Userlevel Sockets Layer over Virtual Interface Architecture. In Proc. IEEE International Conference on Cluster Computing, 2001.

[13] Lawrence Berkeley National Laboratory. MVICH: MPI for Virtual Interface Architecture. http://www.nersc.gov/ research/FTG/mvich/index.html, August 2001. 
[14] L. Liss, Y. Birk, and A. Schuster. Efficient Exploitation of Kernel Access to Infiniband: A Software DSM Example. In Hot Interconnects 11, Stanford University, Palo Alto, CA, August 2003.

[15] J. Liu, B. Chandrasekaran, J. Wu, W. Jiang, S. Kini, W. Yu, D. Buntinas, P. Wyckoff, and D. K. Panda. Performance Comparison of MPI Implementations over InfiniBand, Myrinet and Quadrics. In SuperComputing 2003 (SC '03), November 2003.

[16] J. Liu, J. Wu, S. P. Kini, P. Wyckoff, and D. K. Panda. High Performance RDMA-Based MPI Implementation over InfiniBand. In 17th Annual ACM International Conference on Supercomputing (ICS '03), June 2003.

[17] Message Passing Interface Forum. MPI-2: Extensions to the Message-Passing Interface. Technical Report, University of Tennessee, Knoxville, 1996.

[18] Myricom. MPICH-GM. http://www.myri.com/myrinet/ performance/MPICH-GM/index.html.

[19] Myricom. Socket over GM. http://www.myri.com/scs/ index.html.

[20] F. Petrini, W. Feng, A. Hoisie, S. Coll, and E. Frachtenberg. The Quadrics Network: High-Performance Clustering Technology. IEEE Micro, 22(1):46-57, 2002.

[21] R. Gupta, P. Balaji, D. K. Panda, and J. Nieplocha. Efficient Collective Operations using Remote Memory Operations on VIA-Based Clusters. In Int'l Parallel and Distributed Processing Symposium (IPDPS ’03), April 2003.

[22] W. Rehm, R. Grabner, F. Mietke, T. Mehlan, and C. Siebert. Development of an MPICH2-CH3 Device for InfiniBand. http://www.tu-chemnitz.de/informatik/RA/cocgrid/ Infiniband/pmwiki.php/InfiniBandProject/ProjectPage.

[23] M. Snir, S. Otto, S. Huss-Lederman, D. Walker, and J. Dongarra. MPI-The Complete Reference. Volume 1 - The MPI-1 Core, 2nd edition. The MIT Press, 1998.

[24] H. Tezuka, F. O'Carroll, A. Hori, and Y. Ishikawa. Pin-down Cache: A Virtual Memory Management Technique for Zerocopy Communication. In Proc. 12th International Parallel Processing Symposium (IPPS), March 1998.

[25] R. F. V. D. Wijngaart. NAS Parallel Benchmarks, Version 2.4. Technical Report NAS-02-007, NASA Advanced Supercomputing (NAS), October 2002. 
The submitted manuscript has been created in part by the University of Chicago as Operator of Argonne National Laboratory ("Argonne") under Contract No. W-31-109-ENG-38 with the U.S. Department of Energy. The U.S. Government retains for itself, and others acting on its behalf, a paid-up, nonexclusive, irrevocable worldwide license in said article to reproduce, prepare derivative works, distribute copies to the public, and perform publicly and display publicly, by or on behalf of the Government. 